outcome of a research project" (Royal College of Psychiatrists, 1998). However, many trainees find it difficult to undertake a significant research project, and the experience of a failed attempt to achieve publication can be demoralising.

It is important to distinguish between actually doing research, and appraising and applying the results of research in everyday clinical practice. There is a need to shift the emphasis away from publication as the solitary goal of research time. The CTC suggests that clear individual objectives should be set for the use of a trainee's research time, allowing far greater flexibility over the methods by which these are met. Such objectives may include the ability to formulate answerable questions from clinical situations, to confidently appraise research findings, and to use research evidence in developing service provision or evaluating clinical practice. Documented training objectives allow trainees to monitor their own progress and, in conjunction with their research tutor or supervisor, to ensure that their individual needs are appropriately met during higher training.

While undertaking a research project is one way of meeting these aims, other specific training experiences have an important role. Courses and seminars, clearly focused smaller projects (including audit) and other forms of scholarship can enable trainees to reach the consultant level with a clear grasp of the skills needed to inform and improve their practice. The CTC still believes that the research day forms a crucial part of a fully balanced training but needs to be used more effectively and in an individually tailored manner.

RAMCHANDANI, P., CORBY, C., GUEST, L., et al (2000) The Place and Purpose of Research Training for Specialist Registrars: A View from the Collegiate Trainees' Committee (CTC) www.rcpsych.ac.uk/ members/ctc.htm

ROYAL COLLEGE OF PSYCHIATRISTS (1998) Higher Specialist Training Handbook. Occasional Paper OP43. London: Royal College of Psychiatrists.

*Ed Day Specialist Registrar and CTC Chairman, Paul Ramchandani Specialist Registrar and Research Day Working Party Chairman, Royal College of Psychiatrists, 17 Belgrave Square, London SW1X 8PG

\section{Acquisition of skills during higher specialist training}

Sir: Many skills have to be assimilated during training to equip psychiatrists for the role of consultant. A survey was carried out in 1995 (Haddad \& Creed, 1996) in the North-West Region among newly appointed consultants in general and old age psychiatry. While consultants in their cohort felt senior registrar (SR) training had adequately trained them in areas of clinical, research, teaching and group work they felt poorly trained in areas of general management, personal management and information technology. As a result of this survey trainers were encouraged to use the poorly rated skills as a menu of topics for discussion in supervision, the deficit areas were used as themes for SR training days and SRs were encouraged to attend management training courses.

The survey was recently repeated using the same methodology. Newly appointed consultants in old age and general adult psychiatry who had trained on the Manchester specialist registrar rotation and were appointed between 1995 and 1999 were contacted. Twenty-three consultants out of a total of $33(70 \%)$ completed the questionnaire. Of the 14 (out of 39) skills rated as poorly prepared, 11 of these were in areas of general management, personal management, working with groups and information technology. Newly appointed consultants also felt less than moderately well prepared in three specific clinical areas: (a) use of cognitive-behavioural techniques; ( $b$ ) dealing with patient/relative complaints; and (c) giving evidence in court. These three clinical areas had also been rated poorly in the 1995 survey. Of the 14 skills rated as being 'poor' in the 1995 survey 12 of these remained rated as poor.

Despite the introduction of several changes as a result of the previous survey of newly appointed consultants to the training scheme it was evident that there had been little change in the pattern of response. While training courses no doubt play an important part in training it was clear that the areas where consultants felt most confident were areas where they were likely to have had most practical exposure in training. It maybe that the old medical adage 'see one, do one, teach one' has as much relevance to learning management skills as to learning clinical skills. More novel, 'hands on' learning experiences need to be developed to address these areas of perceived deficit.

HADDAD, P. \& CREED, F. (1996) Skills training for senior registrars. Results of a survey of newly appointed consultants. Psychiatric Bulletin, 203, 391-394.

PLUMMER, D. (1994) Objectives for higher psychiatric training. Working document. London: Royal College of Psychiatrists.

Kate F. Lovett Specialist Registrar in General Adult Psychiatry, Wonford House Hospital, Dryden Road, Exeter EX2 5AF

\section{The wisdom of Ali G}

Sir: Why do Hickling \& Hutchinson (Psychiatric Bulletin, March 2000, 24, 94-95) insist on naming what is essentially a delusion of racial identity "roast breadfruit psychosis"? Not only is the term offensive, akin to having named it "bounty bar psychosis", but as Hari Maharaja's erudite response (Psychiatric Bulletin, March 2000, 24, 96-97) pointed out they have simply "extrapolated a cultural concept - into a diseased state".

Confusion over identity is not unique to Black people and racial identity is only one aspect of one's identity that individuals and groups in society struggle to define. In our complex multi-cultural society 'White' culture is increasingly aware of, exposed to, and influenced by 'non-White'culture. We now have'trustafarians' and comedic characters such as Ali G. A trustafarian is a derogatory term used to describeWhite teenagers who have both trust funds and Rastafarian hairstyles! Ali $\mathrm{G}$ is a comedic caricature of aWhite man mimicking Black $\mathrm{rap} /$ reggae street style and is the invention of aWhite Jewish comedian.

Ali $\mathrm{G}$ became famous after a series of televised spoof interviews with prominent people in the public eye. The interviewees did not realise that Ali G was not the real thing and answered his increasingly ridiculous questions in a naïve, serious or patronising manner. I believe Hickling \& Hutchinson have been taken in by a similar spoof - they have decided to analyse the delusional content of individuals with psychosis and in so doing have revealed more about themselves than any new insight into psychotic illness.

Masum Khwaja Specialist Registrar, The Gordon Hospital, Bloomburg Street, London SW1V 2RH; masumkhwaja@hotmail.com

\section{The 'Hitchcock factor' in advertising}

Sir: I would be interested in the College's view on pharmaceutical advertising in relation to the current campaign to reduce stigma in mental illness. I have recently been struck by the increasing use of negative images by some of the atypical neuroleptic manufacturers.

The black and white images with stark lighting and plenty of shadow seem designed to provoke feelings of fear and menace. Photographing patients as gaunt almost lupine individuals with sunken eyes, angular cheekbones, long hair and humourless, irritated expressions all seem to emphasise the perceived 'differentness' and threat of people with severe mental illness. Likewise 'case histories' laced with suggestions of suicide or danger to emotive groups such as children alongside pictures of a young woman mutilating herself or a frightened girl huddling behind her mother seem to play on misunderstanding and prejudice about schizophrenia.

The risks of alienation, marginalising and stigmatising people suffering with a severe mental illness by using adverts to 
shock and raise fears about dangerousness seem clear. It is particularly concerning when they occur in journals aimed at a wide variety of health professionals in a way likely to reinforce preexisting negative stereotypes about the mentally ill.

Mental health care organisations are striving to take on board high quality risk management and evidence based strategies in planning care for the severely mentally ill and advertising gimmicks that owe more to Poe and Hitchcock than to an informed balanced view of mental illness appeal to the irrational antithesis of this approach. It is not much of a partnership if drug companies use adverts that would cause distress to anyone suffering from schizophrenia or members of their families.

C. J. Haley Locum Consultant Psychiatrist, Primary Care Mental HealthTeam, University Hospital Aintree, 46 Moss Lane, Liverpool L9 8AL

\section{Urinary detection of olanzapine - an aid to compliance confirmed}

Sir: The recent introduction of this technique (Coates, 1999) has led to an important development in psychiatric practice in our area, with possible national consequences.

At a recent mental health review tribunal, due to the fact that a patient had consistently tested negative for olanzapine it was accepted that the patient was not adherent with treatment, contrary to their assertion. The presence of objective evidence of nonadherence was, I believe, instrumental in the tribunal's decision to continue a detention order. Therefore, this shows that there are considerable implications of regular testing for olanzapine, and possibly other antipsychotics, in urine. Within the context of judicial or semijudicial processes, objectively testable evidence will, I believe, always have a greater weight than that of subjective opinion. I therefore believe that urinary detection of olanzapine has been confirmed as an aid to adherence. The issue of whether adherence then becomes compulsion is something that will require further consideration.

COATES, J. (1999) Urinary detection of olanzapine an aid to compliance. British Journal of Psychiatry, 175, 591-592.

John W. Coates Consultant Psychiatrist, Rotherham General Hospital, Moorgate Road, Rotherham, South Yorkshire S60 2UD

\section{Pre-registration training period in psychiatry}

Sir: There may be benefit in the approval and funding of pre-registration posts in specialities other than general medicine and surgery. Indeed a number of posts in general practice have existed for some time.

A recent report by the Royal College of Physicians of London (2000), published on-line, describes "shortening formal training of medical students to four years, and extending pre-registration training to two years", and that the "senior house officer grade should be better linked to opportunities for training in primary care".

These suggestions may also be useful for psychiatric practice, where an increasing throughput of patients, and the need for senior house officer educational time, are putting pressure on services. A pre-registration period spent working in psychiatry should be of training value to those pursuing a career in many different specialities, and closer liaison with general practice in senior house officer training helped by the formation of primary care trusts.

ROYAL COLLEGE OF PHYSICIANS OF LONDON (2000) Hospital Doctors Under Pressure: New Roles for the Health CareWorkforce. http:// www.rcplondon.ac.uk/pubs/wp.hdup.htm

Stephen Carey Specialist Registrar in Psychiatry, Crighton Royal Hospital, Dumfries DG1 4TG: stephencarey@rathcar.freeserve.co.uk

\section{Histories and mental states: from standard formats to standard forms?}

Sir: Kareem \& Ashby (Psychiatric Bulletin, March 2000, 24, 109-110) moot the possibility of a definite Mental State Examination (MSE) format in order to decrease the chances of important items being unexamined or unrecorded. This seems reasonable, but there may be difficulties as our experience auditing alcohol history-taking in the elderly shows.

We audited the alcohol histories recorded for all the elderly patients in Fair Mile Hospital. We found 147 new assessments. Of these, 81 (55\%) failed to record an alcohol history, 37 (25\%) recorded a qualitative history and only $29(20 \%)$ recorded the number of units drunk. Where a history proforma was used the alcohol histories were better. We developed a pro forma for the history and MSE, which included a reminder to record units of alcohol. Having piloted the pro forma, it was introduced for general use and, following educational sessions devoted to alcohol problems in the elderly, notes were re-audited six months later.

In the second audit, when the pro forma was used, only $11 \%$ failed to record an alcohol history, whereas 33\% recorded qualitative and $55 \%$ quantitative histories. This was a statistically significant improvement $\left(P<0.02 ; \chi^{2}\right.$ test with Yates's correction, d.f. $=1)$. But the pro forma was used in only nine out of 74 new assessments! Overall the alcohol history-taking had not improved $(P<0.5$; $\chi^{2}$ test, d.f. $=2$ )

Part of the problem was simply logistical: the pro forma was not readily available. But there was also a reluctance to use it. There was a concern that it made history-taking and the MSE less natural. Of course, such a pro forma does not have to be slavishly completed at the time of the history and MSE. Nevertheless, there are important issues involved in the use of such a pro forma which are worthy of further consideration.

*Julian C. Hughes Consultant Psychiatrist, Gibside Unit, Centre for the Health of the Elderly, Newcastle General Hospital, Westgate Road, Newcastle uponTyne NE4 6BE, Jonathan M. Griffiths Senior House Officer, Jaap van der Boom Senior House Officer. Formerly of the Department of Old Age Psychiatry, Fair Mile Hospital, Cholsey, Oxfordshire 